\title{
SMALL-AMPLITUDE INTERNAL WAVES DUE TO AN OSCILLATORY PRESSURE*
}

\author{
By N. C. MAHANTI** (Visva-Bharati University, Santiniketan 731235, W. Bengal)
}

\begin{abstract}
The initial-value problem of waves in superposed fluids which are otherwise unlimited due to an axisymmetrical oscillatory pressure is solved by the method of integral transforms. The wave integral representing interfacial displacement is evaluated in an asymptotic form that remains uniformly valid through the transition zone.
\end{abstract}

1. Introduction. In the theory of wave-generation in a fluid, both homogeneous and nonhomogeneous, initially at rest, due to an oscillatory pressure (Stoker [1], Wehausen and Laitone [2], Sen [3], Debnath [4, 5]), one comes across an integral representing surface (or interfacial) displacement whose integrand possesses pole and stationary point and is bounded at the pole. This type of integral is evaluated asymptotically by the above authors in their respective papers with the help of some well-known methods. The asymptotic expression of the integral in each case takes different values for different positions of the stationary point relative to the pole and becomes singular when stationary point coincides with the pole. This leads us to seek an asymptotic solution of the problem that remains uniformly valid even when the stationary point coincides with the pole. In an earlier paper considering the initial-value problem of waves due to an axisymmetrical pressure acting on the surface of an infinitely deep fluid, the author [6] has presented a uniformly valid asymptotic solution by the method of van der Waerden [7]. In this paper, we wish to take up the corresponding problem in superposed fluids with a view to presenting the same type of solution by the same method as used in [6].

We note that the solution of our earlier paper [6] may be recovered from that here by letting $\rho^{\prime}=\left(\rho_{1}-\rho_{2}\right) /\left(\rho_{1}+\rho_{2}\right)=1$ i.e. $\rho_{2}=0, \rho_{1}$ and $\rho_{2}$ being the densities of lower and upper fluids. The article ends with the derivation of two limiting cases of interest and the asymptotic envelope of the progressive wave travelling at the interface of the fluids.

2. Formulation and integral solution of the problem. We suppose that a fluid of density $\rho_{2}$ occupying the upper half-space $(y>0)$ lies in stable equilibrium over another fluid of density $\rho_{1}>\rho_{2}$ occupying the lower half-space $(y<0)$. Evidently, the interface between the fluids is defined by the equation $y=0$. The $x$-and $z$-axes of the rectangular coordinate system lie on the undisturbed interface. We would like to study the three-dimensional axisymmetrical linearized wave motion consequent upon the application of a pressure $f(\gamma)$ $\exp (i \omega t) H(t), H(t)=$ Heaviside unit function, at the interface.

The equation together with the attendant boundary and initial conditions of the linearized wave motion are as follows:

$$
\varphi_{j \gamma \gamma}+\gamma^{-1} \varphi_{j \gamma}+\varphi_{j y y}=0, \quad j=1,2
$$

* Received June 16, 1978. The author wishes to thank Professor A. R. Sen of Visva-Bharati University for guidance in the preparation of this paper. This work has been supported in part by a Post-Doctoral Research Fellowship of CSIR, New Delhi.

** Present address: I.I.T., Hauz Khas, New Delhi 110029. 
where $0 \leq \gamma<\infty, 0 \leq y<\infty$ when $j=2$ and $-\infty<y \leq 0$ when $j=1$;

$$
\begin{gathered}
\varphi_{1 y}=\varphi_{2 y} \quad \text { on } y=0 ; \\
\rho_{1}\left(\varphi_{1 t t}+g \varphi_{1 y}\right)=\rho_{2}\left(\varphi_{2 t}+g \varphi_{2 y}\right)+i \omega f(\gamma) \exp (i \omega t) \quad \text { on } y=0 ; \\
g\left(\rho_{1}-\rho_{2}\right) \zeta=\rho_{1} \varphi_{1 t}-\rho_{2} \varphi_{2 t}-f(\gamma) \exp (i \omega t) \quad \text { on } y=0 ; \\
\varphi_{1} \rightarrow 0 \quad \text { as } y \rightarrow-\infty, \varphi_{2} \rightarrow 0 \quad \text { as } y \rightarrow+\infty ; \\
\varphi_{1}=\varphi_{2}=\zeta=0 \quad \text { at } t=0 ; \\
\left(\gamma \varphi_{j \gamma}, \gamma \varphi_{j}\right) \rightarrow 0 \quad \text { as } \quad \gamma \rightarrow 0, \infty ;
\end{gathered}
$$

where $\varphi_{1}$ and $\varphi_{2}$ are velocity potentials of the wave motion in the lower and upper fluids, $\zeta$ is the wave elevation at the interface and the suffixes other than $j, 1,2$ denote partial derivation.

The above system of equations may be solved by the zero-order Hankel transform in $\gamma$; the zero-order Hankel transform $\bar{F}(k)$ of $F(\gamma)$ is defined as

$$
\bar{F}(k)=\int_{0}^{\infty} \gamma F(\gamma) J_{0}(k \gamma) d \gamma .
$$

By applying the transform (2.7) and solving and governing equations, the integral solution for the displacement $\zeta$ at the common boundary between the fluids may be written as

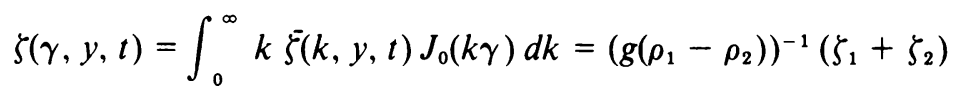

where

$$
\begin{aligned}
& \zeta_{1}=\int_{0}^{\infty} k f(k) \frac{(\sigma / 2)(\sigma+\omega) \exp (i \sigma t)-\sigma^{2} \exp (i \omega t)}{\sigma^{2}-\omega^{2}} J_{0}(k \gamma) d k, \\
& \zeta_{2}=\int_{0}^{\infty} k \bar{f}(k)(\sigma / 2)(\sigma+\omega)^{-1} J_{0}(k \gamma) \exp (-i \sigma t) d k, \\
& \sigma^{2}=g k / \rho^{\prime}, \quad \rho^{\prime}=\left(\rho_{1}-\rho_{2}\right) /\left(\rho_{1}+\rho_{2}\right),
\end{aligned}
$$

and $k$ is the wave number.

3. Asymptotic analysis of $\zeta_{1}$ and $\zeta_{2}$. In this section, we wish to evaluate $\zeta_{1}$ and $\zeta_{2}$ in an asymptotic form for large $\gamma$ and $t$ correct to terms of order $O\left(\gamma^{-1}\right)$ by the method of van der Waerden and the stationary-phase principle.

For $k \gamma \gg 1$,

$$
J_{0}(k \gamma) \sim(2 \pi k \gamma)^{-1 / 2}[\exp (i(k \gamma-\pi / 4))+\exp (-i(k \gamma-\pi / 4))] .
$$

By substituting (3.1) in (2.9) and (2.10) and applying the stationary-phase principle, $\zeta_{1}$ and $\zeta_{2}$ are reduced as follows:

$$
\begin{aligned}
\zeta_{1} \sim & \frac{\exp (i \pi / 4)}{(2 \pi \gamma)^{1 / 2}} \int_{0}^{\infty} k^{1 / 2} f(k) \frac{(\sigma / 2)(\sigma+\omega) \exp (i \sigma t)-\sigma^{2} \exp (i \omega t)}{\sigma^{2}-\omega^{2}} \\
& \cdot \exp (-i k \gamma) d k+O\left(\gamma^{-3 / 2}\right), \gamma \rightarrow \infty \\
\zeta_{2} \sim & \frac{\exp (-i \pi / 4)}{(2 \pi \gamma)^{1 / 2}} f_{0}^{\infty} k^{1 / 2} f(k)(\sigma / 2)(\sigma+\omega)^{-1} \exp (i(k \gamma-\sigma t)) d k+O\left(\gamma^{-3 / 2}\right), \gamma \rightarrow \infty
\end{aligned}
$$


We note that the integrand of (3.2) becomes $0 / 0$ at the pole $\sigma=\omega$, i.e. at $k=\omega^{2} \rho^{\prime} / g$, and the phase $\sigma t-k \gamma$ is stationary at $k=g t^{2} / 4 \gamma^{2} \rho^{\prime}$. This leads us to seek a uniform asymptotic expansion of $\zeta_{1}$ in the neighborhood of $\gamma=g t / 2 \omega \rho^{\prime}$ which brings together the pole and the stationary point. To this purpose, the method of van der Waerden is outlined below.

Let $k=\rho^{\prime} \sigma^{2} / g$. Then $\zeta_{1}$ assumes the form

$$
\begin{gathered}
\zeta_{1} \sim \frac{\exp (i \pi / 4)}{(2 \pi \gamma)^{1 / 2}}\left(\rho^{\prime} / g\right)^{3 / 2}\left[\int_{0}^{\infty} \frac{\sigma^{3} \bar{f}\left(\rho^{\prime} \sigma^{2} / g\right)}{\sigma-\omega} \exp \left\{i\left(\sigma t-\sigma^{2} \rho^{\prime} \gamma / g\right)\right\} d \sigma\right. \\
\left.-2 \exp (i \omega t) f_{0}^{\infty} \frac{\sigma^{4} \bar{f}\left(\sigma^{2} \rho^{\prime} / g\right)}{\sigma^{2}-\omega^{2}} \exp \left(-i \rho^{\prime} \sigma^{2} \gamma / g\right) d \sigma\right],
\end{gathered}
$$

where the path of integration is shown in Fig. 1 for $\omega>g t / 2 \gamma \rho^{\prime}$ and in Fig. 2 for $\omega<g t /$ $2 \gamma \rho^{\prime}$. It can be easily verified that the values of the integrals on the circular quadrant and along the imaginary axis vanish as $R \rightarrow \infty$. Integrating along this path, we obtain the second integral of (3.4) as

$$
\sim-\frac{\pi i}{2} \omega^{3} \bar{f}\left(\omega^{2} \rho^{\prime} / g\right) \exp \left(-i \rho^{\prime} \omega^{2} \gamma / g\right)
$$

for $\omega^{2} \rho^{\prime} \gamma / g \gg 1$. In the first integral of (3.4), we put the new variable

$$
u=\sigma / \omega-g t / 2 \omega \rho^{\prime} \gamma .
$$

Then the integral is reduced to

$$
f_{0}^{\infty}\left[\sigma^{3} \bar{f}\left(\sigma^{2} \rho^{\prime} / g\right)(\sigma-\omega)^{-1} \frac{d \sigma}{d u}\right] \quad \exp \left\{i\left(g t^{2} / 4 \gamma \rho^{\prime}-\omega^{2} u^{2} \rho^{\prime} \gamma / g\right)\right\} d u
$$

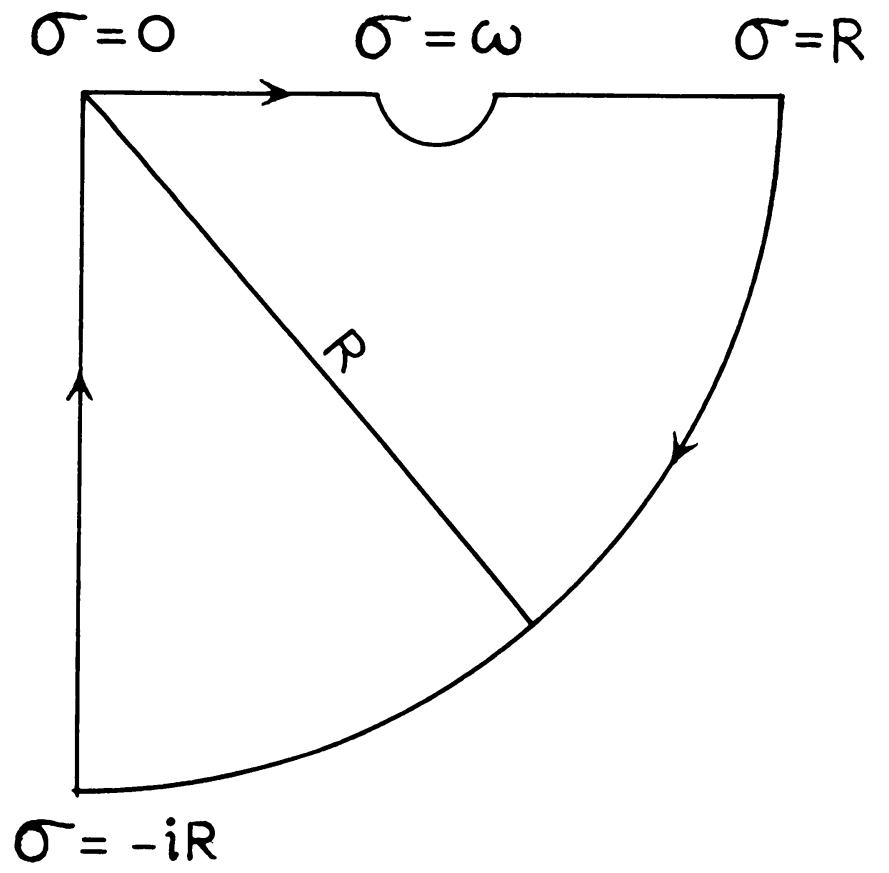

FIG. 1 


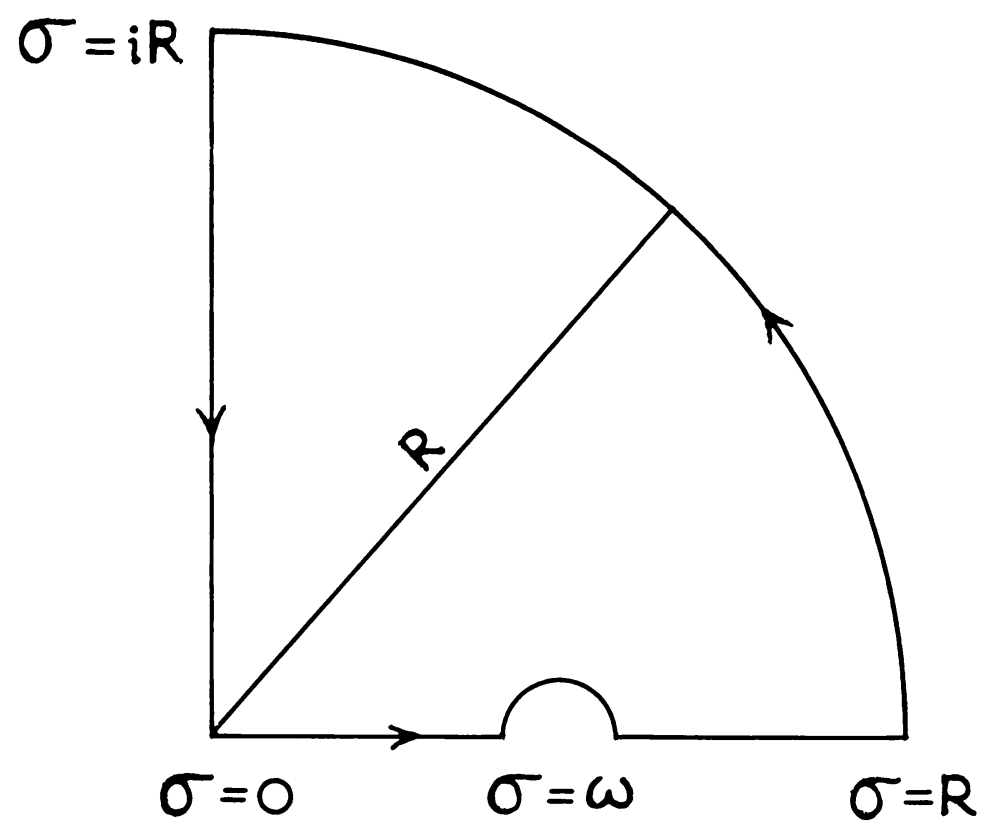

FIG. 2

Let us write

$$
\sigma^{3} \bar{f}\left(\sigma^{2} \rho^{\prime} / g\right)(\sigma-\omega)^{-1} \frac{d \sigma}{d u}=\left(u-u_{0}\right)^{-1} A+\sum_{0}^{\infty} B_{n} u^{n}
$$

where $u_{0}=1-g t / 2 \omega \rho^{\prime} \gamma$. The constants $A$ and $B_{0}$ are found as

$$
A=\omega^{3} \bar{f}\left(\omega^{2} \rho^{\prime} / g\right), \quad B_{0}=\frac{\omega}{g t / 2 \gamma \rho^{\prime}-\omega}\left[g^{3} t^{3} / 8 \gamma^{3} \rho^{\prime 3} \bar{f}\left(g t^{2} / 4 \gamma^{2} \rho^{\prime}\right)-\omega^{3} \bar{f}\left(\omega^{2} \rho^{\prime} / g\right)\right] .
$$

Substituting (3.8) in (3.7) and then applying Watson's lemma to (3.7), we find that (3.7) assumes the form

$$
\begin{aligned}
& A \exp \left(i g t^{2} / 4 \gamma \rho^{\prime}\right) f_{-\infty}^{\infty} \frac{\exp \left(-i \gamma \omega^{2} u^{2} \rho^{\prime} / g\right)}{u-u_{0}} d u \\
& +B_{0} \exp \left(i g t^{2} / 4 \gamma \rho^{\prime}\right) f_{-\infty}^{\infty} \exp \left(-i \gamma \omega^{2} u^{2} \rho^{\prime} / g\right) d u
\end{aligned}
$$

The extension of the limits of the integrals in (3.9) introduces an error of terms of order $O\left(\gamma^{-1}\right)$. Evaluating the integrals in (3.9), we find that (3.9) is asymptotically equivalent to

where

$$
\begin{aligned}
& -\pi \sqrt{ } 2 A \exp \left\{i\left(\omega t-\omega^{2} \gamma \rho^{\prime} / g+\pi / 4\right)\right\} \operatorname{ci}\left\{\frac{\omega^{2} \gamma \rho^{\prime} u_{0}{ }^{2}}{g}\right) \\
& \cdot \operatorname{sgn} u_{0}+\left(\frac{\pi g}{\gamma}\right)^{1 / 2} B_{0} \omega^{-1} \exp \left\{i\left(g t^{2} / 4 \gamma \rho^{\prime}-\pi / 4\right)\right\}
\end{aligned}
$$

$$
\operatorname{cis}(x)=c(x)+i s(x)=\int_{0}^{x} \frac{\exp (i t)}{(2 \pi t)^{1 / 2}} d t .
$$


Introduction of (3.10) and (3.5) in (3.4) lead finally to

$$
\begin{aligned}
& \zeta_{1} \sim \frac{i \sqrt{ } \pi \rho^{\prime 3 / 2} \omega^{3} \bar{f}\left(\omega^{2} \rho^{\prime} / g\right)}{g^{3 / 2}(2 \gamma)^{1 / 2}}\left\{1+i(1+i) c i s\left(\frac{\omega^{2} \rho^{\prime} \gamma u_{0}{ }^{2}}{g}\right) \operatorname{sgn} u_{0}\right\} \\
& \cdot \exp \left\{i\left(\omega t-\omega^{2} \rho^{\prime} \gamma / g+\pi / 4\right)\right\}+\frac{\rho^{\prime 3 / 2} B_{0}}{2^{1 / 2} \omega g \gamma} \exp \left(i g t^{2} / 4 \gamma \rho^{\prime}\right)+O\left(\gamma^{-3 / 2}\right), \quad \gamma \gg 1 .
\end{aligned}
$$

By again applying the method of stationary-phase, the asymptotic value of $\zeta_{2}$ may be written as

$$
\zeta_{2} \sim \frac{g^{3} t^{3} \bar{f}\left(g t^{2} / 4 \gamma^{2} \rho^{\prime}\right)}{8(2)^{1 / 2} \gamma^{4}\left(\omega+g t / 2 \gamma \rho^{\prime}\right)} \exp \left(-i g t^{2} / 4 \gamma \rho^{\prime}\right)+O\left(\gamma^{-3 / 2}\right), \quad \gamma \gg 1 .
$$

Eq. (2.8), taken with (3.11) and (3.12), now gives the complete asymptotic behavior of $\zeta$.

4. Limiting cases. (a) When $g t \ll 2 \omega \rho^{\prime} \gamma, u_{0}>0$. Since $\operatorname{cis}\left(\left(\omega^{2} \gamma \rho^{\prime} / g\right) u_{0}{ }^{2}\right) \sim 2^{-1 / 2} \exp$ $(i \pi / 4)\left(\right.$ see [8]), it is easy to see from (3.11) that the dominant term of $\zeta_{1}$ vanishes.

(b) When $g t \gg 2 \omega \rho^{\prime} \gamma, u_{0}<0$. Proceeding as above, we find that the dominant term of $\zeta_{1}$ is reduced to

$$
\frac{(2 \pi)^{1 / 2} i \rho^{\prime 3 / 2} \omega^{3} \bar{f}\left(\omega^{2} \rho^{\prime} / g\right)}{g^{3 / 2} \sqrt{ } \gamma} \exp \left\{i\left(\omega t-\omega^{2} \rho^{\prime} \gamma / g+\pi / 4\right)\right\}
$$

5. Asymptotic envelope. If $\bar{f}\left(\omega^{2} \rho^{\prime} / g\right) \neq 0$, the dominant progressive wave (3.11) has the envelope

$$
\frac{\pi^{1 / 2} \rho^{\prime 3 / 2} \omega^{3} \bar{f}\left(\omega^{2} \rho^{\prime} / g\right)}{g^{3 / 2}(2 \gamma)^{1 / 2}} R(U)
$$

where

$$
\begin{gathered}
R(U)=\left(P_{1}{ }^{2}+P_{2}{ }^{2}\right)^{1 / 2}, \\
P_{1}+i P_{2}=1+i(1+i) c i s\left(\frac{1}{2} \pi U^{2}\right) \operatorname{sgn} u_{0}, \quad U=\frac{g t-2 \omega \rho^{\prime} \gamma}{\left(2 \rho^{\prime} \gamma \pi g\right)^{1 / 2}}
\end{gathered}
$$

and the phase angle is $\tan ^{-1}\left(P_{2} / P_{1}\right)$. Following Miles [9], we may illustrate graphically the time-wise distribution of $R(U)$.

6. Physical conclusions. The wave motion at the interface may be divided into two distinct components $\zeta_{1}$ and $\zeta_{2}$ having different characteristics. The first component $\zeta_{1}$ is uniform in the neighbourhood of $\gamma=g t / 2 \omega \rho^{\prime}$. The non-uniformity of second component $\zeta_{2}$ does not arise.

The results of this paper are in perfect agreement with those of our earlier paper [6] in the sense that the results of [6] may be recovered from those here by letting $\rho^{\prime}=1$, i.e. $\rho_{2}=$ 0 .

We note that the dominant wave is a progressive wave expressed in terms of Fresnel integral. This wave moves with the velocity $g / \omega \rho^{\prime}$.

The two values of group velocities $d \sigma / d k$ at the stationary point $k=g t^{2} / 4 \gamma^{2} \rho^{\prime}$ and at the pole $k=\omega^{2} \rho^{\prime} / g$ are respectively equal to $\gamma / t$ and $g / 2 \omega \rho^{\prime}$. The circular region $\gamma=(g /$ $\left.2 \omega \rho^{\prime}\right)^{t}$ which brings together the pole and stationary point advances with the group velocity $g / 2 \omega \rho^{\prime}$. 
The limiting case (a) shows that the progressive wave disappears and the wave motion is wholly dispersive at places for which $\gamma \gg g t / 2 \omega \rho^{\prime}$.

\section{REFERENCES}

[1] J. J. Stoker, Water waves, New York, Interscience (1957)

[2] J. V. Wehausen and E. V. Laitone, Surface waves, in Handbuch der Physik IX, Springer, Berlin (1960)

[3] A. R. Sen, Deep-water surface waves due to arbitrary periodic pressures, Proc. Nat. Inst. Sci. India 28, 612-631 (1962)

[4] L. Debnath, An asymptotic treatment of the transient development of axisymmetric surface waves, Appl. Sci. Res. 21, 24-36 (1969)

[5] L. Debnath, On transient development of internal waves in stratified ocean, Indian J. Pure Appl. Math. 1, 372388 (1970)

[6] N. C. Mahanti, Small amplitude surface waves due to an oscillatory pressure, Israel J. Tech. 15(3) (1978) (to appear)

[7] B. L. van der Waerden, On the method of saddle points, Appl. Sci. Res. 2B, 33-45 (1951)

[8] A. Erdélyi et al., Higher transcendental functions, 2, New York, McGraw-Hill (1953)

[9] J. W. Miles, Transient gravity wave response to an oscillatory pressure. J. Fluid Mech. 13, 145-150 (1962) 